\title{
Microbiology of lower respiratory tract infection in workers of garment industry of Kathmandu
}

\author{
Pant $\mathbf{S}^{1}$, Bhusal KR ${ }^{1}$, Manandhar $\mathbf{S}^{2}$ \\ ${ }^{1,2}$ Lecturer, Kantipur Dental College, Kathmandu University, Dhapasi, Kathmandu, ${ }^{3}$ Lecturer, Department \\ of Microbiology, National College,Tribhuvan University, Khusibu, Kathmandu
}

\section{ABSTRACT}

\section{OBJECTIVES}

This study was designed with the objectives of describing the distribution pattern of microorganisms responsible for causing LRTI in the workers of garment industries.

\section{MATERIALS AND METHODS}

A total of 198 cases of suspected person of Lower Respiratory Tract infection (LRTI) LRTI were included in this study. This study was conducted between November 2009 to April 2010. Specimen for the study was expectorated sputum. Gram-stain, Ziehl-Neelsen stains and culture were performed.

\section{RESULTS}

On direct microscopic examination, $20.51 \%$ were Gram positive bacteria, $79.48 \%$ were Gram negative bacteria and $4 \%$ were smear positive AFB. On culture sensitivity examination, $22 \%$ percent showed growth of different bacteria in different culture media. The bacteria isolated from the samples included Klebsiella pneumoniae (15.38\%), Proteus mirabilis (15.38\%) and Citrobacterfruendii(15.38\%). Gram Negative bacteria were found most susceptible to Ciprofloxacin $(92.30 \%, 24 / 26)$ and Amikacin $(92.30 \%$, 24/26). Similarly, Gram Positive bacteria were found most susceptible to Ciprofloxacin $(100 \%, 8 / 8)$ followed by Cloxacillin and Cephalexin (87.5\%, 7/8). Smear positive AFB was significantly associated with not using the protective measures (mask) by workers and presence of symptoms (cough for more than two weeks, night sweat, hemoptysis and anorexia) ( $p=0.031)$. Culture positivity was significantly associated with symptoms like production of purulent sputum ( $p=0.045)$.

\section{CONCLUSION}

There was insignificant association between LRTI and risk factors present in working room of garment industries. Most of the isolates were sensitive to Ciprofloxacin and resistance to Ampicillin and Cephalexin.

Key Words: Lower respiratory tract infection, Garment industries, Sputum and antibiotic susceptibility 


\section{INTRODUCTION}

Lower respiratory tract infection (LRTI) is defined as the inflammation of the respiratory tract starting from the trachea to the alveoli and subsequent multiplication of an infectious agent. ${ }^{1}$ Predisposing factors for LRTI are malnutrition, breast feeding and vitamin A deficiency, increased frequency of low birth weight and prematurity, smoking and alcohols, viral infections, high-risk age groups (children younger than 5 years and adults older than 55-65 years), air pollution and occupational exposure host-related risk factors such as immunosuppression, diabetes, drug therapy, conditions associated with decreased pulmonary clearance functions like asthma, chronic bronchitis, chronic obstructive pulmonary disease etc. ${ }^{2}$ Common causes of community acquired LRTI include Streptococcus pneumoniae, Haemophilusinfluenzae, Mycoplasma pneumoniae, Moraxella catarrhalis, Chlamydia spp, Legionellaspp, Staphylococcus aureus, Klebsiella pneumoniae, Mycobacterium spp,viruses and fungi. ${ }^{3}$

Occupational diseases are usually defined as diseases arising out of or in the course of employment. They may be grouped as diseases due to physical agents, chemical agents and biological agents. ${ }^{2}$ Among the many types of industries present in Nepal, the manufacturing of carpet is presently leading and occupying $54 \%$ of the total export commodity. Untrained manpower, substandard machinery, lack of quality control, lack of health awareness among the workers has direct impact on health of the workers. Frequent respiratory tract infection, skin diseases, sexually transmitted diseases and industrial accidents are some of the health problems noted among the workers of garment industry. People exposed to organic dusts may have a high prevalence of respiratory tract symptoms which are related to dust exposures and smoking habits. ${ }^{4}$

Acute respiratory infections (ARI) are one of the most important causes of morbidity and mortality throughout the world. Globally, about 4.2 million ALRI deaths are estimated to occur among all age groups. ${ }^{5}$ According to the report of Ministry of Health, Government of Nepal, $3.4 \%$ of morbidity is caused by acute RTI. Although occupational safety and health is very important issue at an individual, social and national level, it has not received much attention so far in Nepal. Therefore present study has been designed with an objective to describe distribution pattern and risk factors of LRTI in workers population of garment industries of Kathmandu. Furthermore,antimicrobial susceptibility testing of bacterial isolates has also been described. The study possibly highlights importance of screening of LRTI in workers of garment industry.

\section{METHODS}

It was a case control study comprising 198 cases of garment factory workers carried from November 2009 to April 2010. This study was conducted to find out the bacteriological profile and antibiotic susceptibility pattern of isolated bacteria. The study populations were the persons working in seven different garment industries of Kathmandu Valley. Samples were allocated to each center according to population proportionate to its size. We assigned an identification number to the study population starting from one, in an increasing order. First respondent was selected randomly in each center. The other participants were selected in a systematic manner, excluding two persons who lied in consecutive order between two respondents. Consent was taken from individuals for study. All individuals with or without complaint of lower respiratory tract infections were selected purposefully from garment 
industries. Information was recorded, by interviewing through a structured questionnaire. Working environment was observed for ventilation, presence of air pollution and adequate passage of light etc were recorded in structured questionnaire. Expectorated morning sputum of individuals was taken as specimen. Triplicate specimen were collected from each individual. Laboratory methods included were macroscopic examination, Microscopic examinations, Cultural examinations, Ziehl-Neelsen staining technique and Antibiotic susceptibility testing. Culture was done on 5\% Blood agar (BA), 5\% chocolate agar, Mac Conkeyagar and Nutrient agar (NA).The isolated bacteria were identified using morphological, microscopy and biochemical tests following standard procedures. Bile solubility and optochin sensitivity tests were performed for the suspected pneumococcal colonies from blood agar. For identification of $H$. influenza, Satellitism Test and identification discs having $\mathrm{X}$ and $\mathrm{V}$ factor were used. Finally, data management and analysis was done using SPSS 16 version.

\section{RESULTS}

From total study population (198), 39.53\% (17/43) of males and $60.46 \%$ (26/43) of females were sputum culture positive. Workers of age below twenty years showed highest percentage $(41.86 \%)$ of culture positivity. Different 11 types of bacteria were isolated (table 1), giving presence rate of 19.69\% (39/198). $79.48 \%$ (31/39) of the organisms isolated were Gram negative bacteria whereas $20.51 \%$ ( 8/39) were Gram positive bacteria. The bacteria isolated from the samples included were Klebsiella pneumoniae (15.38\%), Proteus mirabilis (15.38\%), Citrobacter fruendii (15.38\%), Moraxella catarrhalis (12.82\%),
Streptococcus pneumonia (10.25\%), Staphylococcus aureus (10.25\%), Yersinia spp (5.12\%), Enterobacter spp (5.12\%), Escherichia coli (5.12\%), Pseudomonas spp (2.56\%), and Serratia spp $(2.56 \%)$ in order of ranking. Haemophilus influenzae were not found in the study population. Study population taken for the study were found to have the clinical history like chest pain (87), shortness of breath (60), fever (46), prolonged cough (51), sputum production (137), anorexia (61), night sweat (29) and hemoptysis (17). Some of the workers complained about having backache, joint pain, lower abdominal pain, cancer, old history of Tuberculosis and skin diseases. Of the total study population (198), 4\% (8/198) were smear positive AFB and $96 \%(190 /$ 198 ) were smear negative AFB (figure 1) and $37.5 \%$ (3/8) of male and $62.5 \%(5 / 8)$ of female were smear positive AFB. Table 2 shows that smear positive AFB was significantly associated with few symptoms of LRTI (presence of cough for more than two weeks $(\mathrm{p}=0.001)$, anorexia $(\mathrm{p}=0.047)$, night sweat $(\mathrm{p}=0.000)$ hemoptysis $(\mathrm{p}=0.000)$ and not using protective measures mask ( $p=0.031)$ by workers. Insignificant association was obtained with risk factors such as smoking habit $(\mathrm{p}=0.205)$ and alcohol taking habit $(\mathrm{p}=0.823)$, working room environment and educational status of workers $(p=0.145)$. In this study sputum samples obtained from 23 females and 15 male workers were culture positive. Significant association of culture positivity was obtained with symptoms like production of purulent sputum $(\mathrm{p}=0.045)$ and insignificant association was obtained with other symptoms like chest pain ( $\mathrm{p}=0.686)$, Shortness of breath $(p=0.528)$, fever $(p=0.355)$ and cough for more than two weeks $(p=0.373)$. Association of culture positive was insignificant with educational status $(p=0.785)$, working room environment $(p=0.355)$, risk 
factors like smoking habit $(\mathrm{p}=0.089$ and alcohol taking habit ( $\mathrm{p}=0.422$ ) and not use of protective measures mask $(\mathrm{p}=0.649)$ by workers. As shown in table 3, Gram Positive bacteria were found most susceptible to Ciprofloxacin $(100 \%, 8 / 8)$ followed by Cloxacillin and Cephalexin $(87.5 \%, 7 / 8)$, Erythromycin and Ampicillin $(75 \%, 6 / 8)$ respectively. They were least susceptible to Co-trimoxazole $(62.5 \%, 5 / 8)$. As shown in table 4, Gram Negative bacteria were found most susceptible to Ciprofloxacin $(92.30 \%, 24 / 26)$ and Amikacin $(92.30 \%, 24 / 26)$ followed by Gentamicin (82.75\%, 24/29), Ceftazidime (33.33\%, 9/27), Cephalexin $(25 \%, 7 / 28)$ and Ampicillin $(21.42 \%, 6 /$ 28).

Table 1: Distribution of microbial isolates isolated in study population

\begin{tabular}{|c|c|c|c|}
\hline Organisms & $\begin{array}{l}\text { Number } \\
\text { of } \\
\text { cases }\end{array}$ & $\begin{array}{c}\text { Percentage among } \\
\text { corresponding bacterial } \\
\text { isolates }\end{array}$ & $\begin{array}{c}\text { Percentage } \\
\text { among total } \\
\text { isolates }\end{array}$ \\
\hline \multicolumn{4}{|l|}{ Gram Positive Bacteria } \\
\hline Streptococcus pneumoniae & 4 & 50 & 10.25 \\
\hline Staphylococcus aureus & 4 & 50 & 10.25 \\
\hline Total & 8 & 100 & 20.51 \\
\hline \multicolumn{4}{|l|}{ Gram Negative Bacteria } \\
\hline Citrobacter fruendii & 6 & 19.35 & 15.38 \\
\hline Proteus mirabilis & 6 & 19.35 & 15.38 \\
\hline Klebsiella pneumoniae & 6 & 19.35 & 15.38 \\
\hline Moraxella catarrhalis & 5 & 16.12 & 12.82 \\
\hline Yersinia spp & 2 & 6.45 & 5.12 \\
\hline Enterobacter spp & 2 & 6.45 & 5.12 \\
\hline Escherichia coli & 2 & 6.45 & 5.12 \\
\hline Pseudomonas spp. & 1 & 3.22 & 2.56 \\
\hline Serratia spp. & 1 & 3.22 & 2.56 \\
\hline Total & 31 & 100 & 79.48 \\
\hline
\end{tabular}


Figure 1: Percentage of AFB positive and AFB negative in sputum sample of study population.

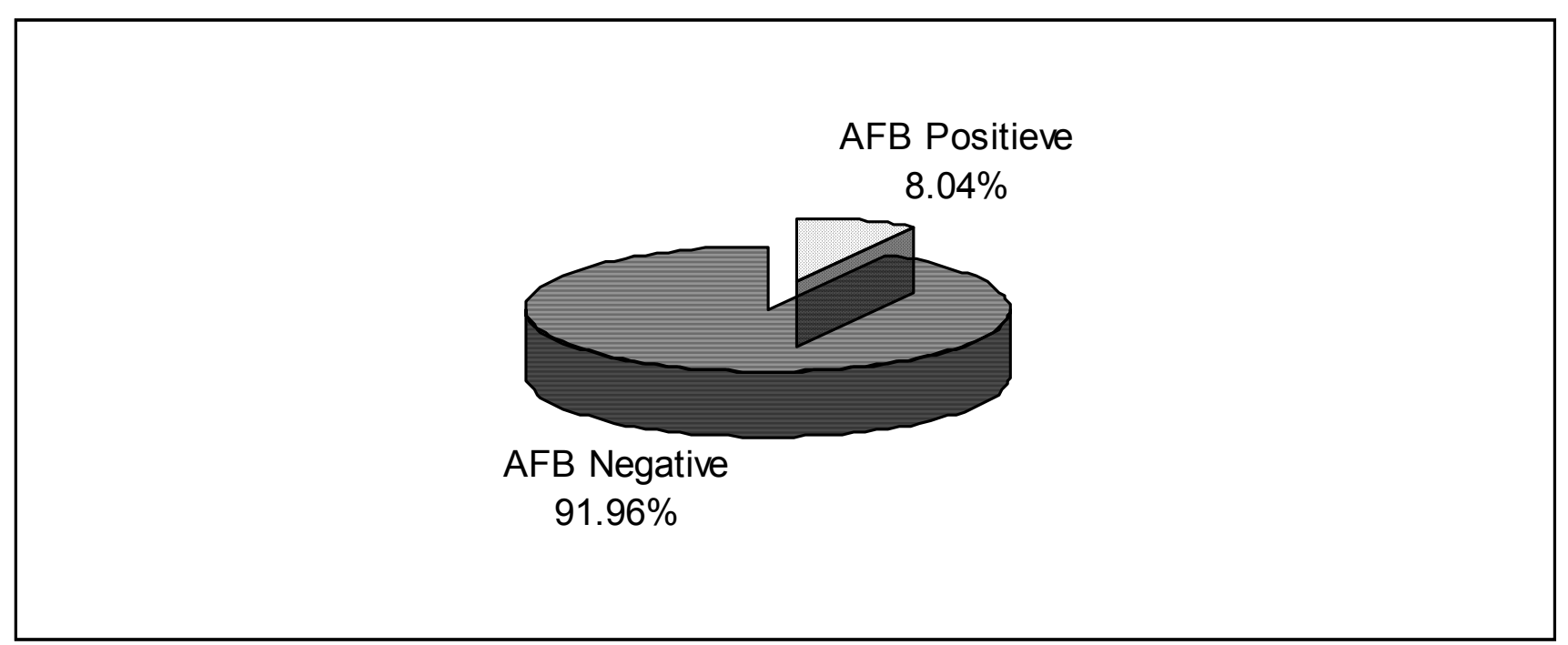

Table 2: Association of smear positive AFB with symptoms of LRTI

\section{Symptoms}

Cough for more than two weeks

$$
\text { Yes }
$$$$
\text { No }
$$

Anorexia

$$
\begin{aligned}
& \text { Yes } \\
& \text { No }
\end{aligned}
$$

Night sweat

$$
\begin{gathered}
\text { Yes } \\
\text { No }
\end{gathered}
$$

Hemoptysis

$$
\begin{gathered}
\text { Yes } \\
\text { No }
\end{gathered}
$$

Productive sputum

$$
\begin{aligned}
& \text { Yes } \\
& \text { No }
\end{aligned}
$$

Chest pain

$$
\begin{aligned}
& \text { Yes } \\
& \text { No }
\end{aligned}
$$

Shortness of breath

Yes

No$$
3
$$$$
5
$$

$$
4
$$$$
4
$$

145

\section{AFB}

Negative

Significance

(p-value)

6

2

5

3

56

0.047

134

6

2

23

0.000

167

13

177

7

130

0.252

1

60

6

81

0.71

2

109

57

133

Fever

Yes 
Table 3: Antibiotic pattern of Gram positive bacteria $(n=8)$

\begin{tabular}{lcccc}
\multicolumn{2}{c}{ Antibiotics } & Sensitive & \multicolumn{2}{c}{ Resistant } \\
\cline { 2 - 2 } & No & \% & No & \% \\
Ciprofloxacin & 6 & 75 & 2 & 0 \\
Cephalexin & 8 & 100 & 0 & 12.5 \\
Co-trimoxazole & 7 & 87.5 & 1 & 37.5 \\
Erythromycin & 5 & 62.5 & 3 & 25 \\
Cloxacillin & 6 & 75 & 2 & 12.5
\end{tabular}

Table 4: Antibiotic sensitivity pattern of Gram negative bacteria

\begin{tabular}{|c|c|c|c|c|c|}
\hline \multirow{2}{*}{ Antibiotics } & \multicolumn{2}{|c|}{ Sensitive } & \multicolumn{2}{|c|}{ Resistant } & \multirow[t]{2}{*}{ Total } \\
\hline & No & $\%$ & No & $\%$ & \\
\hline Ampicillin & 6 & 21.42 & 22 & 78.57 & 28 \\
\hline Ciprofloxacin & 24 & 92.30 & 2 & 7.69 & 26 \\
\hline Cephalexin & 7 & 25 & 21 & 75 & 28 \\
\hline Amikacin & 24 & 92.30 & 2 & 7.69 & 26 \\
\hline Gentamicin & 24 & 82.75 & 5 & 17.24 & 29 \\
\hline Ceftazidime & 9 & 33.33 & 18 & 66.66 & 27 \\
\hline
\end{tabular}




\section{DISCUSSION}

This study was undertaken to find out the bacteriological profile and the sensitivity pattern of the isolates from the workers of garment industries from Kathmandu valley. In this study, respondents complained of gastritis, urinary tract infection, high blood pressure, lower abdominal pain in female, chest pain and breathing problems, mild fever at night, anorexia, hemoptysis, productive cough, body and joint pain, backache, skin diseases. Among these, the complaints like chest pain, fever, productive cough, breathing problem, hemoptysis, and anorexia were characterized as respiratory tract problems and other cases as health problems. Out of total population $22 \%$ were sputum culture positive and $78 \%$ were sputum culture negative. Among total population $39.53 \%$ male and $60.46 \%$ female were sputum culture positive. Of the total population, workers of age below twenty years showed highest percentage $(41.86 \%)$ of culture positivity. According to study done in garment industries of Bangladesh , most of female workers (over 85\% ) suffer from malnutrition, ill health, reproductive health problem, anemia, respiratory problem, urine infection etc. ${ }^{6}$ Culture positivity depends on nature of sputum, transportation time and the number of organism present in the sample. Arora, et al 2001 had obtained growth in $72 \%$ cases, whereas Dalvi et al 1983 obtained growth in $57 \%$ of samples. The yield of sputum culture in this study was low (22\%) comparable to other studies which report a 50\% yield with sputum cultures. ${ }^{7,8}$ Out of the total study population $4 \%$ were AFB positive and $96 \%$ were AFB negative. Among total population $37.5 \%$ male and $62.5 \%$ female were AFB positive. Smear AFB positive were equally obtained (25\%) in age group below 20, 30-39, 5059 years and $12.5 \%$ in age group 20-29, 40-49 years respectively. SmearAFB positive was obtained in more number from female in study population (Table 3 ). This may be because of higher percentage of malnutrition in lower class females in Nepalese context. Eleven different bacteria were isolated, giving a prevalence rate of $19.69 \%$. This consisted of $60.46 \%$ from female workers and $39.53 \%$ from male workers. The bacteria isolated from the samples included $79.48 \%(31 / 39)$ Gram negative bacteria whereas $20.51 \%$ (8/39) were Gram positive bacteria. Incidence of Haemophilusinfluenzae was not found in study population this may be due to delayed transport of sample to laboratory which might have cause death of fastidious organism. Gram Negative Bacteria (79.48\%) outnumbered the growth of Gram Positive Bacteria. Presence of Gram negative bacilli colonizing the oropharynx increases with severity of underlying respiratory alteration. The proportion of Gram-negative bacteria causing LRTIs was $79.48 \%$, which exceeds to the $63.9 \%-68.4 \%$ of a report from Germany. ${ }^{9}$ This study was very much related to the similar study carried by Egbagbe et al., 2006 and Christopher et al., 2010 in which K. pneumoniae (52.5\%) was the most common organisms followed by $S$. aureus $(10 \%)$. This isolation of $K$. pneumoniae as predominant organism also agreed with another study carried by Okesola et al., 2002-2005 and by Navaneeth et al., 2002. This study is in concordance with that of study carried by Eldeev et al., 2006 where K. pneumoniae $(11.27 \%)$ was most prevalent followed by $P$. aeruginosa $(6.26 \%)$ among GNB and $S$. aureus (17.71\%) as the most prevalent. Similar study carried by Hidron et al., 2008 found K. pneumoniae as the most common pathogens. Study done in paper mill industry by Niemela et al., 1985 found that $K$. pneumonia was responsible formost cases of LRTI. This study shows the prevalence of $K$. pneumoniae as the commonest isolate this could be due to reason 
that $K$. pneumoniae is a relatively common cause of pneumonia in persons suffering from alcoholism, as alcohol intake directly inhibits the ability of lung immune cell to kill bacteria. And in this study, most of the workers were alcoholic. The smear positive AFB was insignificantly associated with all symptoms of LRTI. However, AFB was significantly associated with few symptoms of LRTI (presence of cough for more than two weeks [ $p=0.001]$, anorexia $[p=0,047]$, night sweat $[p=0.000]$ and hemoptysis $[p=0.000])$ that were present in the workers. Study carried out by Nesli et al.,1946 reported that one half of the TB patient had developed symptoms like cough, low grade fever, weight loss and fatigue, night sweat, chest pain and one-third of patient had hemoptysis. There was significant as well as insignificant association of sputum culture positivity with symptoms of LRTI in study population. Significant association was obtained in symptom like production of purulent sputum $(\mathrm{p}=$ 0.045 ) and insignificant association was obtained in other symptoms like chest pain, SOB, fever and cough. Smoking appears to be an important risk factor for the acquisition of a lower respiratory tract infection (bronchitis, influenza, pneumonia, and tuberculosis).In our study, smoking habit of study population was insignificantly associated with smear positive AFB and culture positivity. Though statistically association was insignificant but there was no greater difference of smear positive AFB and culture positivity cases in smokers and non-smokers in study population. Mark et al., 1982 found that $57 \%$ women were smoker and they had acute respiratory tract illness associated with smoking. Alcohol consumption is another risk factor for LRTI. Alcohol consumption is known to affect both systemic and pulmonary immunity, predisposing the patient to pulmonary infections. In study done by Thomas et al., 2006 conclude that alcohol abuse was associated with an increased risk of LRTI. In our study, most of the respondents were alcoholic and there was insignificant association between alcohol consumption and smear positive AFB and culture positivity. By the use of preventive measures, risk of much communicable disease can be minimized. In this study, very few workers had taken preventive measures to avoid occupational dust exposures. Of the total study population, only 4 workers had used personal protective measures (Mask). Smear positive AFB was obtained in more number in workers not using protective measures mask in working place. But the association was statistically insignificant between sputum culture positivity and use of protective measures. Today, antibiotics remain the front-line therapy for conquering bacterial infections. Antimicrobial agents have provided the way for both physicians and the public perceive bacterial infections and their treatment. Different antibiotics are useful for antibacterial therapy. The therapy is guided by antibiotic susceptibility pattern of isolates. Quinolones were found to be most effective antibiotic against both GNB and GPB. S. pneumoniae isolates showed sensitivity to penicillins, macrolides and quinolones. In this study, most of the isolates were sensitive to the quinolones (ciprofloxacin), but poorly sensitive to ampicillin and Cephalexin.High antimicrobial resistances to ampicillin and penicillin have also been reported in France, Germany and Japan. ${ }^{10}$ The high rate of resistance to Ampicillin, Ceftazidime, Cephalexin, Amikacin observed in this study may reflect the fact that these are the most commonly prescribed antibiotics in the hospital and also the most easily available for community without prescription. Thus from our study it becomes clear that antimicrobial resistance has become significant public health problem even in our set up. It is wise to control this situation before it takes a deadly shape. Following the 
recommendation given by summit on antimicrobial resistance, like not indulging patient demands for untreated infection, educating patients about the proper use of antibiotics, identifying the pathogen, choosing short -course, narrow -spectrum antibiotics, completing the full course of therapy, encouraging patients to get vaccinated, improving resistance surveillance systems, will definitely help controlling this situation. Through the efforts by clinicians, microbiologist, patients and public health officials, the antimicrobial resistance can be slowed. In this way, maintenance and prolonged efficacy of existing drugs can be obtained.

\section{CONCLUSION}

Lower respiratory tract infection was seen in workers of garment industries of Kathmandu. Presence of LRTI was found more in female workers than male workers. Workers of age below 20 years showed much prevalence towards LRTI. K. pneumoniae was mostly predominant organism. There was insignificant association between LRTI and risk factors present in working room of garment industries. Smoking and alcohol taking habits of workers were also insignificantly associated with LRTI. But association was significant between smear positive AFB and not using the protective measures (mask). Most of the isolates were sensitive to Ciprofloxacin and resistance to Ampicillin and Cephalexin.

\section{ACKNOWLEDGEMENTS}

We would like to thank the garment workers and staffs of garment industries of Kathmandu.

\section{REFERENCES}

1. MacFarlane J, Holmes W, Gard P, et al . Prospective study of the incidence, aetiology and outcome of adult lower respiratory tract illness in the community. Thorax 2001;56:109-114.

2. Park. K.Parks, Occupational health, Text book of Prevalence and social medicine, $19^{\text {th }}$ edi., Jabalpur, India :M/s Banarsids Bhanot publishers 2007:658-673.

4. Simpson JC, Niven RM, Pickering CA, et al. Prevalence and predictors of work related respiratory symptoms in workers exposed to organic dusts 2007;8-10.

5. World Health Organization. The global burden of disease: 2004 update. Geneva: World Health Organization 2008.

6. Akhter S, Salahuddin AFM, Iqba M et al, Health and occupational safety for female workforce of Garment industries in Bangladesh, Journal of Mechanical Engineering 2010;41:(1) 66-68.

7. File TM. A review of community acquired pneumonia. Infect Dis ClinPract 1996;5:127-135.

8. Bartlett JG. Community-acquired pneumonia in adults: Guidelines for management of the Infectious Diseases, Society of America. Clin Infect Dis 1998;26: 811-838.

9. Kohlenberg A, Schwab F, Geffers C. Time trends for Gram-negative and multidrug resistance Gram positive bacteria associated with nosocomial infections in German intensive care units between 2000-2005. Clin Microbial Infect 2008;14: 93-96.

10. Schito GC, Debbia EA, Marchese A, et al, The evolving threat of antibiotic resistance in Europe: new data from the Alexaner Profect, J Antimicrob Chemother 2000;46:3-9. 